\title{
Obstructed viscoplastic flow in a Hele-Shaw cell
}

\author{
Masoud Daneshi, ${ }^{1}$ Jordan MacKenzie, ${ }^{1}$ Neil J. Balmforth, ${ }^{2}$ D. Mark Martinez, ${ }^{1}$ \\ and Duncan R. Hewitt ${ }^{3}{ }^{3}$ \\ ${ }^{1}$ Department of Chemical and Biological Engineering, University of British Columbia, \\ Vancouver, Canada V6T $1 Z 4$ \\ ${ }^{2}$ Department of Mathematics, University of British Columbia, Vancouver, Canada V6T 1Z4 \\ ${ }^{3}$ Department of Applied Mathematics and Theoretical Physics, University of Cambridge, \\ Cambridge CB3 OWA, United Kingdom
}

(Received 4 July 2018; revised manuscript received 23 October 2018; published 6 January 2020)

\begin{abstract}
Experiments are conducted exploring the flow of Carbopol past obstacles in a narrow slot and compared with predictions of a model based on the Herschel-Bulkley constitutive law and the conventional Hele-Shaw approximation. Although Carbopol is often assumed to be a relatively simple yield-stress fluid, the flow pattern around an obstacle markedly lacks the fore-aft symmetry expected theoretically. Such asymmetry has been observed previously for viscoplastic flows past obstacles in unconfined geometries, but the narrowness of the Hele-Shaw cell ensures that the stress state is very different, placing further constraints on the underlying origin. The asymmetry is robust, as demonstrated by varying the shape and number of the obstacles, the surfaces of the cell walls, and the steadiness of the flow rate. The results suggest that rheological hysteresis near the yield point may be the cause of the asymmetry.
\end{abstract}

DOI: 10.1103/PhysRevFluids.5.013301

\section{INTRODUCTION}

Slow viscous flow around an obstacle is a classical problem in fluid mechanics, illustrating the so-called Stokes paradox and its resolution. When placed into the confines of a narrow slot-a Hele-Shaw cell-the flow problem becomes (at leading order) equivalent to two-dimensional potential flow around an obstacle. For either unconfined or Hele-Shaw flow, the problem has fore-aft symmetry owing to the reversibility of the steady flow field.

Here we are concerned with the generalization of these flow problems to the situation in which a complex fluid flows around an obstacle. In particular, following recent work on soft matter and complex fluids [1,2], we address the problem of viscoplastic flow around an obstacle in a Hele-Shaw cell. The practical applications are widespread, particularly for drilling and fracture problems in the oil and gas industries where unwanted blockages are a key consideration (see, e.g., Refs. [3-5]). Flow around obstacles placed in a Hele-Shaw cell provides the simplest possible idealization of how a spatial nonuniformity in a slender conduit can create such blockages.

Viscoplastic flows around obstacles have previously been considered in unconfined geometries, both theoretically and experimentally (see, e.g., Refs. [6-9]). For the flow around cylinders and spheres, the yield stress ensures that fluid motion becomes localized around the obstacle, alleviating the Stokes paradox without recourse to inertia. Moreover, theory based on the Herschel-Bulkley constitutive law again predicts fore-aft symmetry. In detail, the flow pattern consists of a "bubble" of yielded material surrounding the obstacle, with triangular or conical plugs attached to its front and back. By contrast, experiments show the surprising feature that the flow and attached plugs are 
(a)
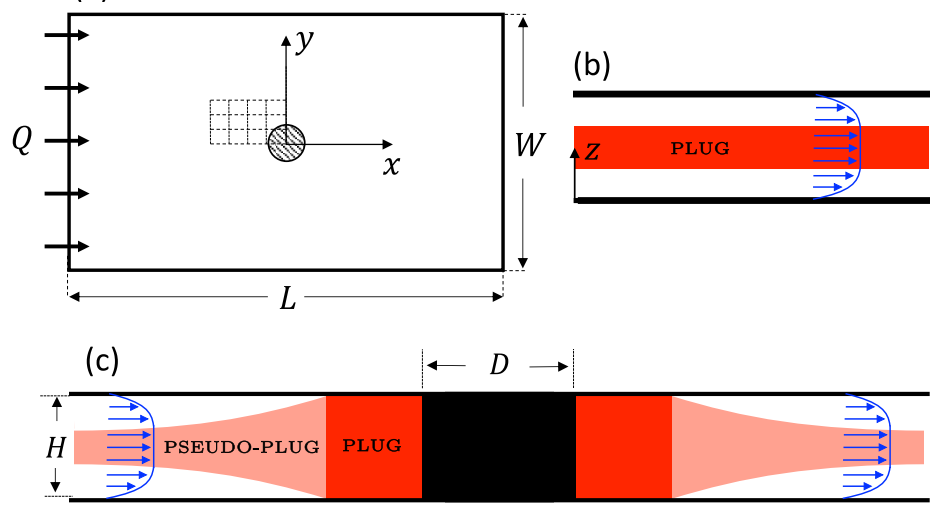

FIG. 1. Viscoplastic flow through a Hele-Shaw cell containing an obstacle. The plane of the cell is shown in (a), where the grid represents the mosaic pattern used to create composite images of the flow field. A velocity profile for flow down a uniform, unobstructed cell, with a central rigid plug, is shown in (b), while the corresponding profile through the centerline of the obstructed cell $(y=0)$ is shown in (c), with a shaded pseudoplug that widens to fill the cell and become truly rigid near the obstacle.

not fore-aft symmetrical. Viscoelastic liquids also display such asymmetry, an effect attributed to the viscoelastic relaxation of the stress [10]. Following on for viscoplastic fluid, nonideal rheological effects such as elasticity or thixotropy have been proposed to explain the asymmetry $[7,11,12]$. Similar asymmetry has been observed in the flow of floating foam rafts around obstacles, which has been modeled as two-dimensional elasto-viscoplastic flow [11].

In a narrow slot [Fig. 1(a)], the situation is very different in two important ways: the flow is forced to yield against the walls in order to move through the slot, and the shear across the slot dominates the strain-rate tensor of the fluid. These differences are well known, and their implications are demonstrated in the expected flow profiles illustrated in Fig. 1. For unidirectional flow down a uniform cell, the profile is characterized by shear layers against the walls, together with a central rigid plug, as shown in Fig. 1(b). When the flow encounters an obstacle in the slot, however, the fluid must slow down and be diverted sideways, preventing the central region from remaining truly rigid. Instead, stresses in the plane of the slot become important to break the plug and hold the central region marginally above the yield stress. The flow profile across the slot remains pluglike, and the central region better referred to as a "pseudoplug" [1,13]. As illustrated in Fig. 1(c), the thickness and speed of the pluglike flow vary along the slot, widening in the vicinity of the obstacle. Eventually, the pseudoplug can grow to fill the cell, at which point the slot becomes spanned by a genuine rigid plug. The distinction between a rigid plug filling the cell and a central, moving pseudoplug bounded by sheared fluid is a key detail of these flows, ensuring that there is no "lubrication paradox" of the sort that has been mistakenly proposed in the past [1].

Confined viscoplastic flow down a narrow slot is therefore rather different than the unconfined flows previously studied; the flow is directed primarily along the slot, rendering it quasi-twodimensional, but the shear occurs mainly across the slot according to a known profile. Thus, unlike in an unconfined geometry where all the velocity gradients must be measured, the velocity in the cell's midplane provides a direct gauge of the key strain rates. In addition, the shear stresses across the slot provide the main resistance to flow, weakening any effects of the extensional stresses, and further simplifying the expected rheological behavior. In other words, the Hele-Shaw cell provides a transparent and definitive setting in which to assess nonideal rheological effects in complex flows (cf. Ref. [14]). 


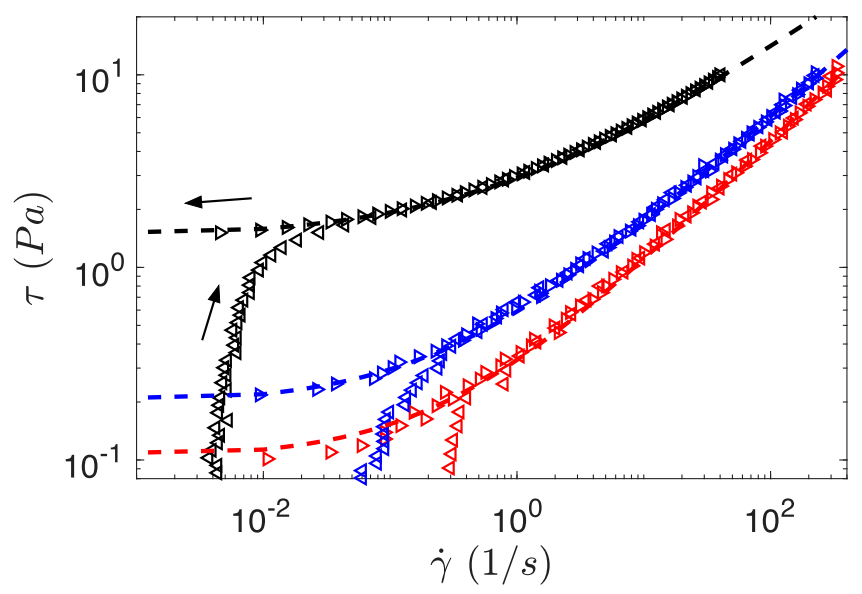

FIG. 2. Flow curves for the three different Carbopol suspensions used in this work [0.075\% (black), $0.06 \%$ (blue), and $0.055 \%$ (red)]. The curves are obtained using a rheometer with roughened parallel plates (MCR501, Anton Paar) and correspond to ramps in shear stress, proceeding first up and then down with a ramp rate of $0.05 \mathrm{~Pa} / \mathrm{s}$. The dashed lines give the Herschel-Bulkley fits in Table I.

The current work follows on from a theoretical study [13] exploring the flow of Bingham fluid around cylindrical obstacles in Hele-Shaw cells. As for the unconfined problem, the theory again predicts that the flow field is fore-aft symmetric. Moreover, the pseudoplugs do indeed expand to fill the cell over confined regions at the front and back of the obstacle. The goals in this paper are to explore the experimental counterpart to this predicted flow structure for a wider array of different kinds of obstacles and to identify any fore-aft asymmetry and constrain its origin. In addition, to provide a more detailed theoretical comparison to these experimental results, we generalize the theory of Ref. [13] to a Herschel-Bulkley fluid and perform further computations (the details of the theoretical formulation and numerical approach can be found in that paper).

\section{EXPERIMENTAL METHOD}

All results reported in this work were obtained in a thin rectangular channel, formed between two acrylic plates (of length $L=105 \mathrm{~mm}$ and width $W=64 \mathrm{~mm}$ ) separated by a height $H=$ $1( \pm 0.05) \mathrm{mm}$; see Fig. 1(a). The flow was generated by a syringe pump delivering a flow rate $Q$ with a maximum of $3 \mathrm{ml} / \mathrm{min}$ (and providing inlet flow velocities of order $10^{-4} \mathrm{~m} / \mathrm{s}$ ). To place obstacles in the cell, we either drilled holes in the plates and inserted a cylinder or three-dimensional printed shapes with the thickness of the channel. The shapes, with cross sections of a circle, square, or stadium (each with a shortest cross-sectional length of $D=11 \pm 0.01 \mathrm{~mm}$ ), were printed with internal compartments to house a magnet, so that the obstacles could be suitably positioned and orientated inside the cell.

The working fluids were three different aqueous suspensions of Carbopol-940. These fluids were well described by the Herschel-Bulkley constitutive law and showed little sign of hysteresis in their measured flow curves above the yield stress (Fig. 2). Near that threshold, the flow curves display the hysteresis commonly found when performing controlled ramps in shear stress, an effect attributable to elastic deformation $[1,15]$. The flow-curve data in Fig. 2 suggest that the fluids do not display any thixotropy of the kind previously found for other Carbopol gels $[16,17]$. Moreover, the fluid was also presheared before injection into the cell, to eliminate the initial nonideal rheological effects reported in sedimenting sphere experiments [7].

The Herschel-Bulkley fits of the yield stress $\tau_{Y}$, consistency $K$ and power-law index $n$ are summarized in Table I. Also listed are measurements of the shear storage and loss moduli, $G^{\prime}$ 
TABLE I. Herschel-Bulkley fits of the Carbopol solutions. Also listed are shear storage and loss moduli $\left(G^{\prime}\right.$ and $G^{\prime \prime}$ ) measurements taken from small amplitude oscillatory rheometry at a frequency of $1 \mathrm{~Hz}$ and a strain amplitude of $\gamma=1 \%$ (below which we confirmed that the two moduli where independent of $\gamma$ ).

\begin{tabular}{lccccc}
\hline \hline $\begin{array}{l}\text { Concentration } \\
(\mathrm{wt} / \mathrm{wt} \%)\end{array}$ & $\tau_{Y}(\mathrm{~Pa})$ & $K\left(\mathrm{~Pa} \mathrm{~s}^{n}\right)$ & $n$ & $\mathrm{G}^{\prime}(\mathrm{Pa})$ & $\mathrm{G}^{\prime \prime}(\mathrm{Pa})$ \\
\hline 0.055 & 0.10 & 0.23 & 0.64 & 3.0 & 0.9 \\
0.060 & 0.20 & 0.39 & 0.57 & 4.2 & 0.7 \\
0.075 & 1.43 & 1.53 & 0.46 & 16 & 2.0 \\
\hline \hline
\end{tabular}

and $G^{\prime \prime}$, from oscillatory rheometry; $G^{\prime}$ is somewhat larger than $G^{\prime \prime}$ for all three fluids, suggesting a linear viscoelastic relaxation time below the yield stress that is less than a second or so [15]. This estimate for the relaxation time was consistent with additional stress relaxation tests and with previous rheometry of Carbopol gels with similar concentration [9].

In addition to these Carbopol suspensions, we also conducted a small number of tests with two others fluids: a glycerol-water mixture and a polyethylene oxide (PEO) solution. The former provides a direct comparison with a Newtonian Hele-Shaw flow, the latter with a prototypical viscoelastic liquid characterized by a relaxation time of a few seconds [18].

Particle-image velocimetry (PIV) was conducted using a swept-field laser-scanning confocal microscope at an imaging frequency of $30 \mathrm{~Hz}$. A $4 \times$-objective lens with working distance of $16 \mathrm{~mm}$ captured the motion of $3 \mu \mathrm{m}$ diameter fluorescent beads, which were seeded at concentration of 0.005 (wt/wt $\%$ ). The position of the observation plane was controlled by a motorized stage and fixed on the central horizontal plane of the cell. Because the field of view of the lens $(2 \mathrm{~mm} \times 2 \mathrm{~mm})$ was too small to capture the whole cell, the full flow field was visualized by stitching together a series of pictures to create a composite image [see Fig. 1(a)]. This procedure limited us to map the full flow field only for a subset of the experiments, and to focus on features at the front or back of the obstacles for most of the tests.

Each experiment was conducted by first cleaning the walls of the cell. In most cases with isolated disks, the walls were also chemically treated to reduce wall slip, as described in Ref. [19]. Carbopol was then pumped through the cell for approximately 20 minutes before flow visualization commenced. Given the flow rate and rheological parameters, we define the Bingham number (the dimensionless strength of the yield stress) by

$$
B=\tau_{Y} K^{-1}\left(W H^{2} / Q\right)^{n} .
$$

To verify the velocity profile across the cell, we undertook some separate studies of flow down a uniform slot with treated walls, combining optical coherence tomography (Thorlabs TEL1300V2BU) with particle-tracking velocimetry [20,21]. Sample profiles are presented in Fig. 3, together with theoretical predictions based on the prescribed flow rate and rheological parameters [13]. These results confirm the removal of any effective slip by the chemical treatment and demonstrate how the theory and experiment are in quantitative agreement. By contrast, and in line with other studies [22], the Carbopol was observed to slip somewhat when the walls were left untreated. As illustrated in Fig. 3, the slip velocity is relatively small (less than about $25 \%$ of the maximum velocity over the range of experimental parameters, as estimated by a linear extrapolation of the particle tracking data to the wall position) with the flow profile largely maintaining the same form as with treated plates.

A related concern was the potentially destructive impact on the wall treatment by the introduction and rearrangement of obstacles placed in the cell, which was achieved by moving magnets and involved dragging the obstacles over the tightly fitting walls. To avoid any possibility that mechanical contact might interfere with the surface treatment, when we explored flows around squares, stadia, or multiple disks, we resorted to the use of cells with untreated walls, notably in reconstructing the full flow field (cf. Fig. 4). Before proceeding down this path, we did, however, verify that, for isolated cylinders inserted through drilled holes in the walls, there were no significant 


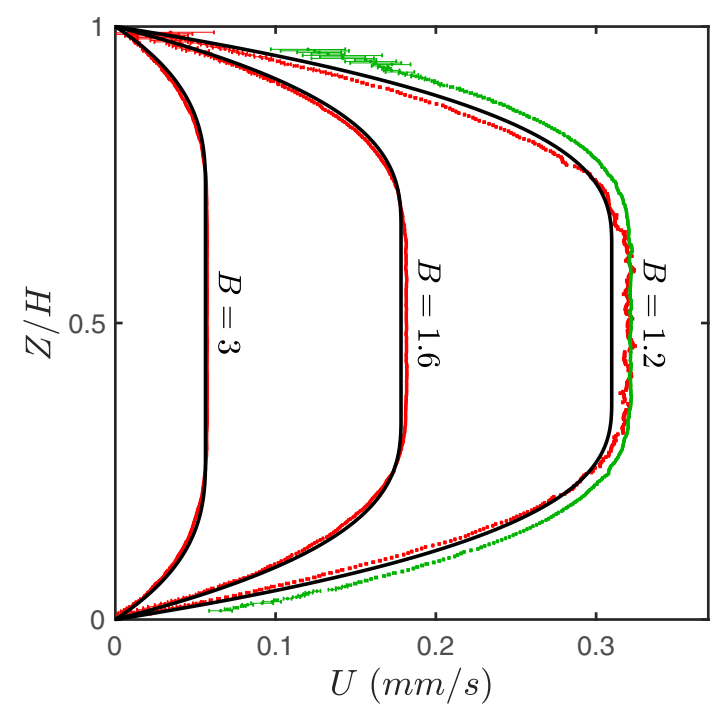

FIG. 3. Experimentally measured velocity profiles for $0.06 \%$ Carbopol in a uniform cell with chemically treated plates at the three Bingham numbers (flow rates) indicated (red). The solid black lines show corresponding theoretical predictions given the fluxes and the rheological parameters in Table I. A profile from a cell with untreated plates for $B=1.2$ (green) is also shown.

differences with results for flows down cells with treated walls [see Fig. 7(c) and the relevant discussion in Sec. III B].

\section{RESULTS}

\section{A. Plug phenomenology}

Sample experimental results for cells with different obstacles are presented in Fig. 4, while a selection of complementary numerical solutions are presented in Fig. 5. Figure 4(a) shows a control experiment in which a Newtonian fluid (a glycerol-water solution) was pumped around a disk. The flow pattern is fore-aft symmetric and compares well with the potential-flow theoretical solution that is also plotted.

The symmetry is preserved when test is repeated with the PEO solution [Fig. 4(b)]. For the unconfined configuration, the flow pattern in such a fluid is expected to become asymmetrical owing to viscoelastic relaxation, which pushes the maximum shear stresses downstream of the top and bottom of the obstacle [10]. For the experiment shown in Fig. 4(b), one might expect that a similar relaxational effect arises and suppresses the plug in the wake. However, no asymmetry is observed for PEO, a result that extends to a range of flow rates and polymer concentrations. On further consideration [14], the absence of fore-aft asymmetry in this viscoelastic flow is not surprising: for the sheared flow in the narrow geometry of our Hele-Shaw cell, the extensional stresses are suppressed and the Weissenberg number is relatively small [typical shear rates are $0.1 \mathrm{~s}^{-1}$, in comparison to the $O(1 \mathrm{~s})$ relaxation time].

Figure 4(c) shows the analogous experiment with Carbopol, which differs in two ways to the Newtonian and PEO tests. First, unyielded plugs spanning the cell appear at the front and rear of the disk, and this feature of the flow is mirrored in the theoretical solutions [Fig. 5(a)]. In detail, however, the structures in the flow field, and in particular the dimensions and shapes of the experimental plugs, differ from their theoretical counterparts. Notably, the experimental plugs have a roughly right-angular form, whereas their theoretical counterparts thin to a pronounced cusplike nose. This discrepancy may arise because the Hele-Shaw theory breaks down on length 


$$
\begin{array}{lll} 
& U / U_{c} & \\
0 & 0.5 X & 0.75 X
\end{array}
$$
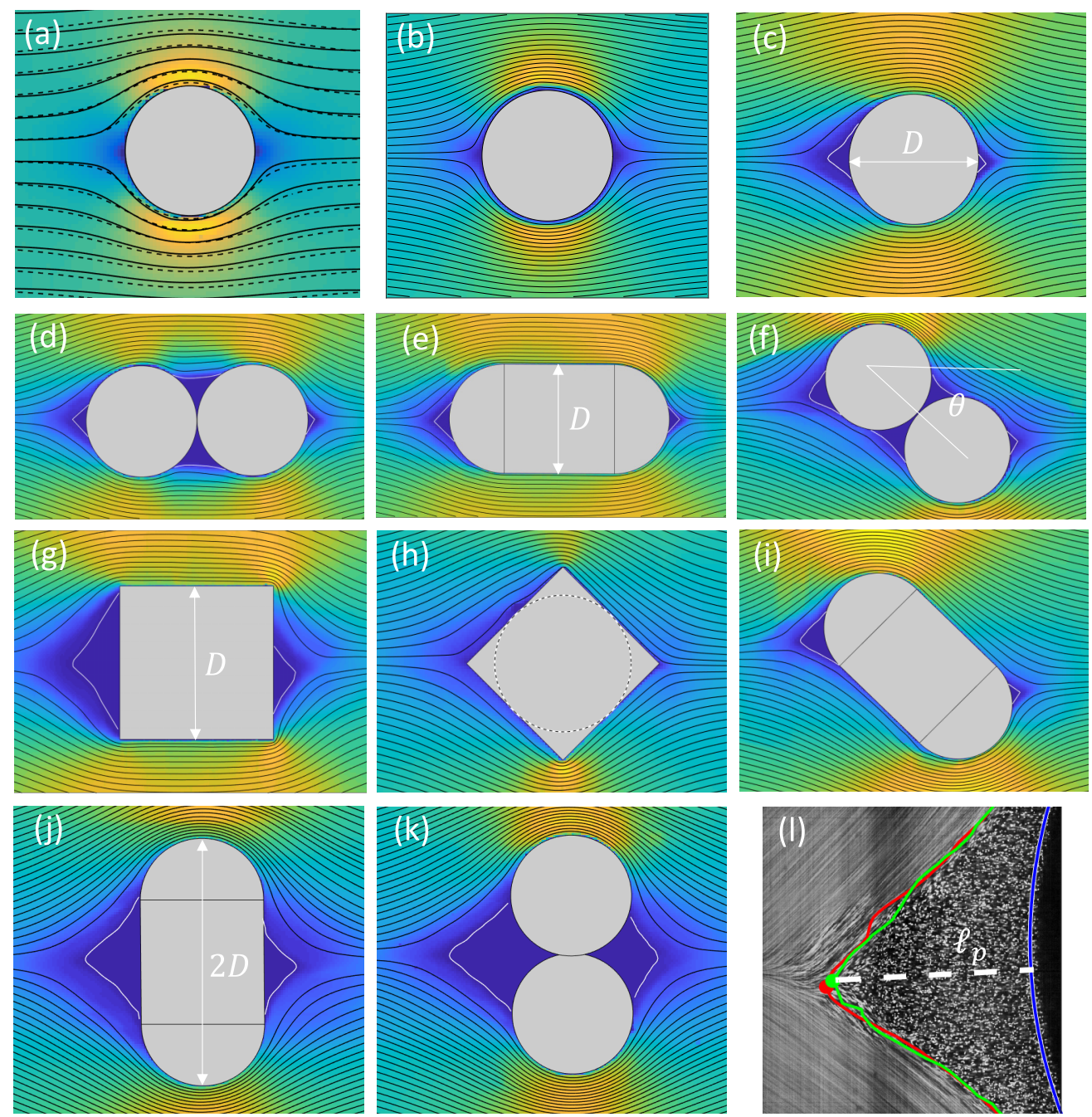

FIG. 4. PIV images showing speed, normalized by the incident speed $U_{c}=Q / W H$, and streamlines (all flowing left to right) along the midplane of the slot around various obstacles. (a) The flow around a disk for a glycerol-water solution ( $31 \mathrm{wt} / \mathrm{wt} \%$ ) at $Q=0.05 \mathrm{ml} / \mathrm{min}$; dashed lines show the corresponding potential-flow solution. (b) Flow field for a viscoelastic PEO solution $(0.75 \mathrm{wt} / \mathrm{wt} \%)$ with $Q=0.16 \mathrm{ml} / \mathrm{min}$. (c)-(k) Flow fields for a Carbopol solution (0.06 (wt/wt\%), untreated walls) with $B=3.1$ (c), (g), (h) and $B=2.7$ (d), (e), (f), (i), (j), (k). The upper limit of the color bar was set at $X=2.8$ for (a), (b), $X=2$ for (j), (k), and $X=1.6$ for (c)-(i). (l) An average image near the plug at the front of a disk $(0.075 \%$ Carbopol solution, $B=2.3$, treated walls). The solid lines indicate the edge of the plug according to edge detection (green) or a velocity threshold (red); the dashed line shows the estimated plug length $\ell_{p}$. White lines in $(\mathrm{c})-(\mathrm{k})$ show the yield surfaces detected using a velocity threshold of $0.0005 \mathrm{~mm} / \mathrm{s}$. 


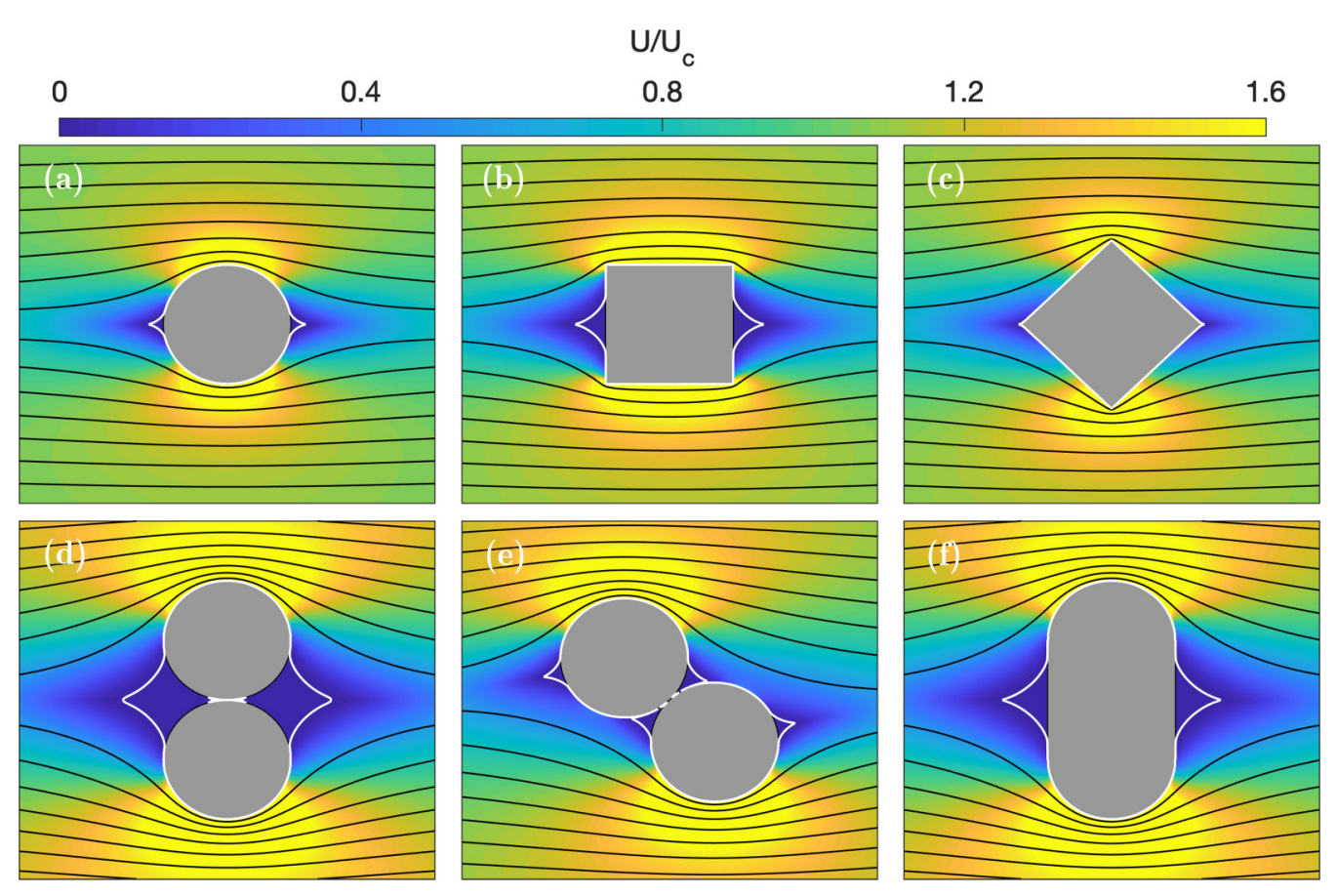

FIG. 5. Speed (shading; scaled by the incident speed $U_{c}$ ) and representative streamlines (solid black lines) along the midplane of the slot for computations of Herschel-Bulkley fluid around the gray-shaded obstacles, for $n=\frac{1}{2}$ and $B=4$. The range of the color bar matches that used in Figs. 4(c)-4(k). The white lines show yield surfaces, given by the threshold $U / U_{c}=1 \times 10^{-3}$.

scales comparable to the height of the slot (here $H \sim 1 \mathrm{~mm}$, which is comparable in size to the discrepancy between theory and experiment) or by the complications in defining the edge of the plug due to undetectable velocities. Such possibilities cannot, however, rationalize the second difference between the Carbopol and earlier tests, which is that there is a marked fore-aft asymmetry in the flow pattern, with the plug at the front being larger than that at the rear, and mirrors findings for unconfined flows $[7,8]$.

Figures 4(d)-4(k) show more experiments with different shapes or combinations of obstacles (all with the $0.06 \%$ Carbopol). Plugs again appear in almost all cases, except when the front or rear of the obstruction has the form of a relatively sharp corner [Fig. 4(h)]. Once again, there is some qualitative agreement with complementary theoretical results for different obstacles [see Figs. 5(b)-5(f)]. However, in every case the experimental flow patterns display a clear fore-aft asymmetry that is absent in the theoretical predictions. There are also quantitative differences with the theoretical predictions for the plugs that are again suggestive of inadequacies in the Hele-Shaw approximation (for example, there are small plugs at the front and back corners of the diamond-shaped obstacle in the computations, but not the experiments).

The limitation of the Hele-Shaw approximation is certainly responsible for the substantially faster flows that arise against the top and bottom of the obstacles in the theoretical solutions; see Fig. 6(a). In particular, in this approximation, fluid is permitted to slide along the boundary of the obstacle, whereas in reality any slip is either much reduced or eliminated entirely, depending on the degree of surface interaction. Consequently, to correct the theory a boundary layer of thickness $H$ is needed that sheathes the obstacle and adjusts the surrounding velocity field to accommodate the true surface condition. The boundary layer is visible in all the experimental images of Fig. 4, as are the excessive high-speed regions in the solutions of Fig. 5. The maximum speeds attained in the theory are therefore too high in comparison to the experiments. In fact, as shown in Fig. 6, because 

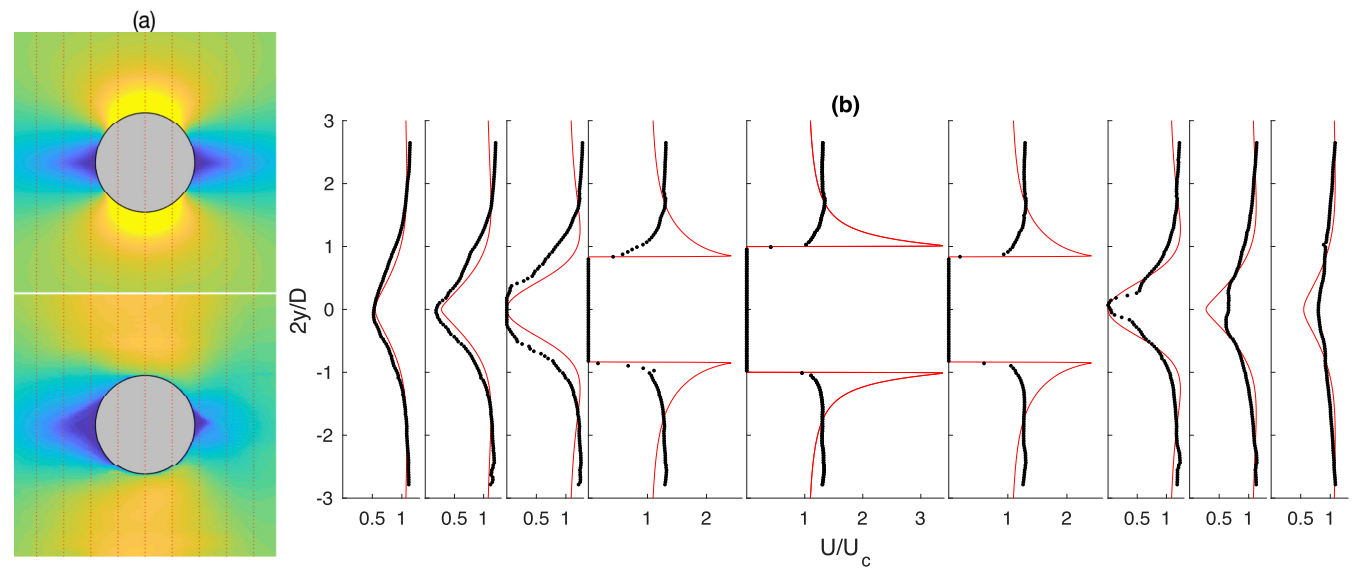

FIG. 6. (a) Scaled theoretical (top) and experimental (bottom) speeds, $U / U_{c}$, for flow around a disk with $n=0.57$ and $B=2.3$, corresponding to panel (1) in Fig. 4 (and using the same scheme for the color map as that figure). In (b), the theoretical (solid lines) and PIV (dots) speeds are plotted along the sections indicated by the vertical dotted lines in (a). The notable disagreement in the velocity profiles past the top and bottom of the disk is a result of a local breakdown of the Hele-Shaw approximation over a distance of the order of the slot thickness from the disk, as discussed in the main text.

the experimental slot is not that narrow $(H / D \approx 0.1)$, the high-speed regions predicted theoretically are almost completely smeared out, rendering the faster flows more distant and broader. Otherwise, the sections of speed across the slot shown in Fig. 6(b) compare fairly well between theory and experiment (modulo the fore-aft asymmetry in the latter).

Note that, for multiple disks and stadia, both the experiments and theoretical computations display a "cloaking" effect similar to that reported in Ref. [23]: when such obstacles are orientated broad-side on, the unyielded plugs at the front and back mask the precise differences in shape, such that the surrounding flow field becomes essentially the same [cf. Figs. 4(j) and 4(k) or Figs. 5(d) and 5(f)]. Only when the yield stress (i.e., $B$ ) is sufficiently small do the plugs fail to cloak the front or back surface, unveiling the true shape differences. [This feature is seen particularly clearly in the theoretical plots of Fig. 8(a) below.] If the broad side of the obstacles is orientated either with the flow or at $45^{\circ}$, no such cloaking effect is possible as the surface of the obstacle is always exposed to unyielded fluid. Nevertheless, in the Hele-Shaw geometry, the yield surface of the plugged-up cavity for the double disks acts somewhat like the straight sides of the stadium, shielding the surrounding flows and rendering similar their patterns [compare panels (d) and (e), or (f) and (i), of Fig. 4].

The Carbopol experiments also display weak symmetry-breaking in the direction transverse to the flow for obstacles with a symmetry plane along the midline of the cell [i.e., up-down symmetry in Fig. 4; compare the magnification of the forward plug in Fig. 4(1) and the speed sections in Fig. 6]. We interpret the broken symmetry to arise from imperfections in the cell geometry and inflow, which apparently become accentuated by the yield stress of the fluid (neither the Newtonian nor viscoelastic experiments were noticeably asymmetrical). These imperfections are not systematic from experiment to experiment, and separate velocimetry measurements across the slot upstream of the obstacle indicate that the incident flow is fully developed.

\section{B. Plug lengths}

To quantify the plug size for the bluff obstacles, we measure the perpendicular distance $\ell_{p}$ from the apex of the triangular plug to the obstacle surface; see Fig. 4(1). This measure is unambiguous and convenient for all but the double disks aligned perpendicular to the flow in Fig. 4(k); for those, we define $\ell_{p}$ as the shortest distance from the apex of the plug to the vertical line drawn 

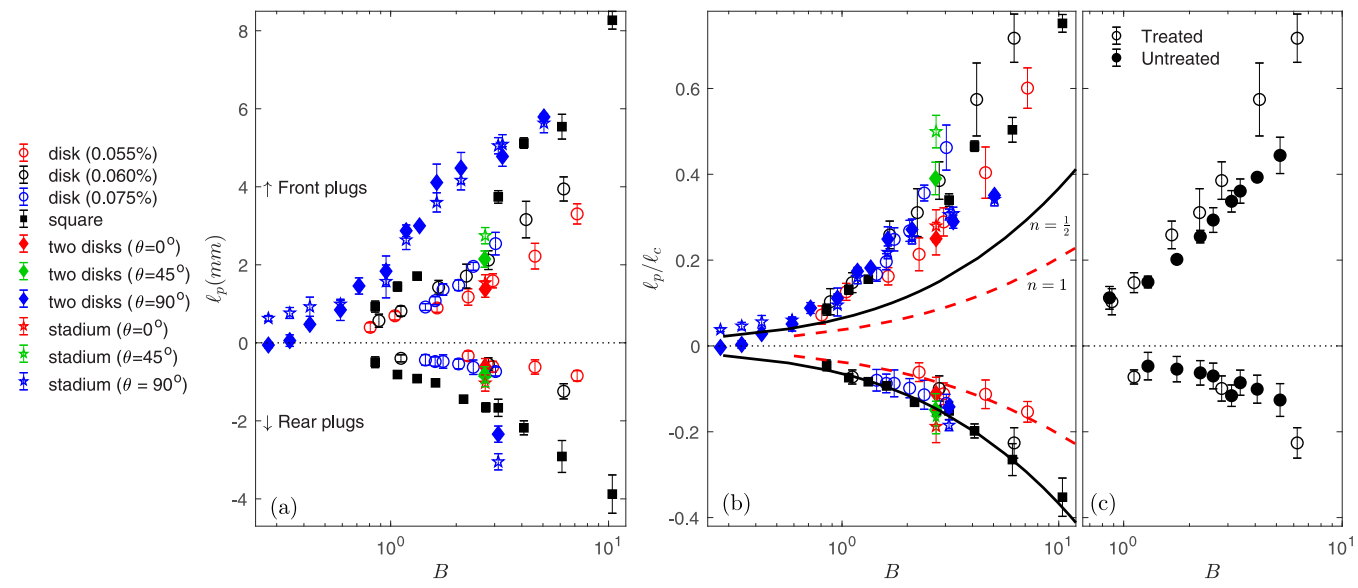

FIG. 7. (a) Dimensional plug lengths $\ell_{p}$ of the configurations shown in Fig. 4, with the conventions indicated by the legend. The walls of the cell are treated to remove slip for the isolated disks, but for the squares, stadia, and multiple disks, the walls are untreated in view of the potentially destructive method of insertion or rearrangement. (b) Scaled plug lengths $\ell_{p} / \ell_{c}$, where $\ell_{c}$ is chosen as indicated in the main text for the three groupings of obstacles. The lines show theoretical results for isolated disks, calculated using the numerical method of Ref. [13] for the Herschel-Bulkley model with $n=0.5$ (black solid) and $n=1$ (red dashed). (c) A comparison of the plug size for flow of $0.06 \%$ Carbopol around disks with treated and untreated acrylic sheets. In all three panels, we plot $\ell_{p}$ as positive for the plugs at the front, and $\ell_{p}<0$ for those at the back. The error bars indicate the standard deviation from three repetitions of each experiment.

between the frontmost or rearmost points of the disks. The plug itself is identified either from edge detection in the average of the images recorded (in which stationary tracer particles appear as bright points whereas moving tracers become blurred into gray streaks) or from a noise-based threshold in the velocimetry. The two methods give comparable results [see Fig. 4(1)]. To further reduce any experimental uncertainty, tests were usually repeated three times, and we report error bars based on the resulting standard deviations which can be as large as $15 \%$.

A systematic survey of plug length as a function of Carbopol concentration, inlet flow rate, and blockage configuration is shown in Fig. 7, with analogous theoretical computations shown in Fig. 8. All the experimental results for disks, squares, and stadia, spanning roughly two decades of Bingham number $(0.1 \leqslant B \leqslant 10)$, are assembled in Fig. 7(a). The fore-aft asymmetry is very clear in these results, with the plug lengths roughly fall into three groups. In particular, the plug lengths for single disks are similar to those of two disks or stadia that are either aligned with or at $45^{\circ}$ to the flow. The plug lengths for double disks or for a stadium aligned perpendicular to the flow are somewhat different but are similar to one another. The square is different again, with a plug length somewhere between those of the other two groups. Although there is no fore-aft asymmetry in the theory, the plug-length data do again fall into the same three groups, with the computations otherwise qualitatively capturing the trend of the experimental measurements. More quantitatively, the prediction of the plug length is somewhat low, curiously aligning more with the data for the rear plugs than those at the front [see Fig. 7(b)].

We achieve some collapse of all the data for the $0.06 \%$ Carbopol by scaling the plug length according to a simple algorithm, which crudely identifies the length of the obstacle over which the incident flow forms an angle greater than $45^{\circ}$ : for obstacles with rounded leading or trailing faces [Figs. 4(c)-4(f) and 4(i)] we scale $\ell_{p}$ with the radius of curvature of the front or rear; i.e., $\ell_{c}=R \equiv D / 2$. For obstacles with a square front or rear face, we scale $\ell_{p}$ by the length of that face; for the square obstacle in Fig. 4(g); this corresponds to a scaling of $\ell_{c}=2 R$. For double disks and stadia aligned perpendicular to the flow [Figs. 4(j)-4(k)], we compose a scaling length from the radius of curvature of the top and bottom of the obstacle plus the square length of the section 

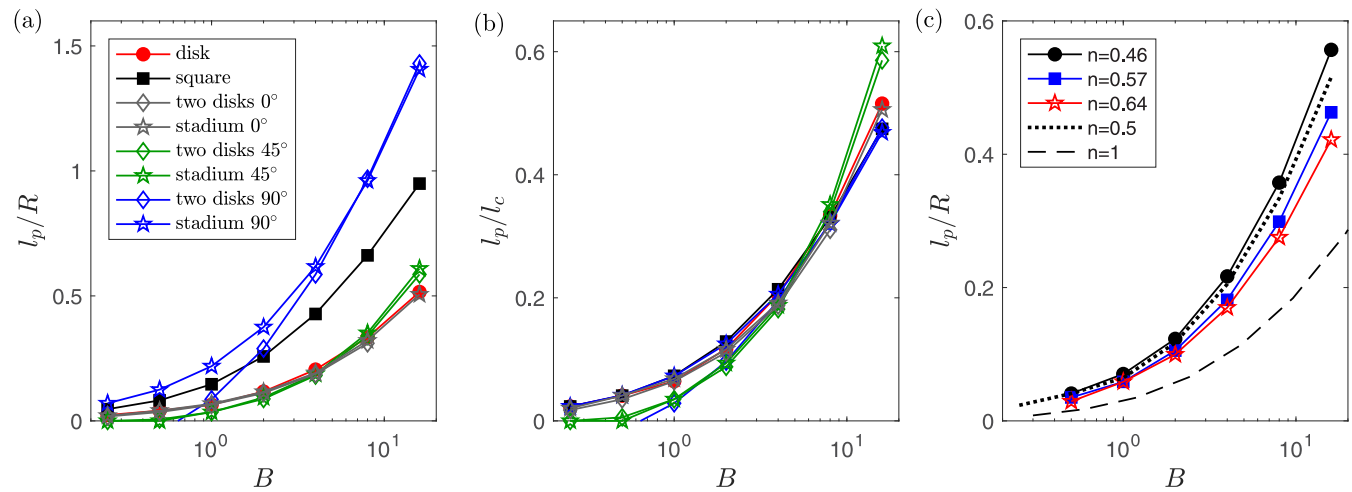

FIG. 8. Theoretical predictions of the plug lengths from numerical simulations for the obstacle arrangements indicated by the legends, and following the experimental results in Fig. 7, showing (a) $\ell_{p} / R$ and (b) $\ell_{p} / \ell_{c}$. These computations have $n=\frac{1}{2}$; in (c) data are shown for isolated disks and the three values of $n$ for the experimental fluids, as well as results for $n=\frac{1}{2}$ and for a Bingham fluid $(n=1)$ [13], for reference.

in between; i.e., $\ell_{c}=3 R$. Figure 7(b) shows the scaled plug lengths, $\ell_{p} / \ell_{c}$. The success of the collapse is reinforced by Fig. 8(b), which performs the same scaling of the theoretical results. The simple algorithm also rationalizes the lack of plugs in the tilted square in Fig. 4(h), where no part of the obstacle is sufficiently inclined against the incident flow.

For different Carbopol concentrations and isolated disks, Figs. 8(a) and 8(b) also indicate that the plug length is fairly insensitive to the power-law index $n$. Nevertheless, at the higher Bingham numbers, the plug lengths for the highest concentration are noticeably lower, suggesting that the plug size may increase with decreasing $n$. Indeed, such a trend is demonstrated by the results of theoretical computations, as shown in Fig. 8(c).

Finally, we report the results of experiments for a single disk in a cell with untreated plates and a range of flow speeds to examine the importance of effective slip. Poumaere et al. [22] have previously suggested that wall slip can create complexity in the flow dynamics down narrow conduits. As illustrated in Fig. 7(c), although there is a small quantitative effect on plug lengths, the degree of fore-aft asymmetry remains unchanged, implying that surface interaction is not the cause of this asymmetry, and that wall slip does not appear to introduce qualitatively different flow dynamics in our Hele-Shaw cells.

\section{Constraining the origin of asymmetry}

To further constrain the origin of the fore-aft asymmetry, we conducted some additional tests. First, we examined steady flow around two disks aligned with the flow, but with varying separations (Fig. 9). The varying separation implies that fluid elements progress along Lagrangian trajectories of different lengths as they deflect around the disks, such that any rheological evolution of fluid elements should be reflected in differences in the overall flow pattern. An increase in the separation distance between the disks leads to no observable effect on either the length of the plug at the front of the first disk or that at the rear of the second disk. Furthermore, once the intervening distance between the plugs is sufficiently long that the stagnant plug bridging the gap has broken up, the front and rear plugs of the second disk are essentially the same length as those of the first. These observations limit the rheological changes that must occur along the streamlines to those that occur over distances of order the diameter of the disks; that is, the distance between the top of the disk and its front or back, which is a few millimeters and corresponds to a typical transit time of 10 or so seconds, given that typical inlet flow speeds are order $10^{-4} \mathrm{~m} / \mathrm{s}$.

Despite this, the data in Fig. 9 demonstrate that the plug bridging the gap can be longer than the combined length of the plugs at the front and back of an isolated disk. The leading 

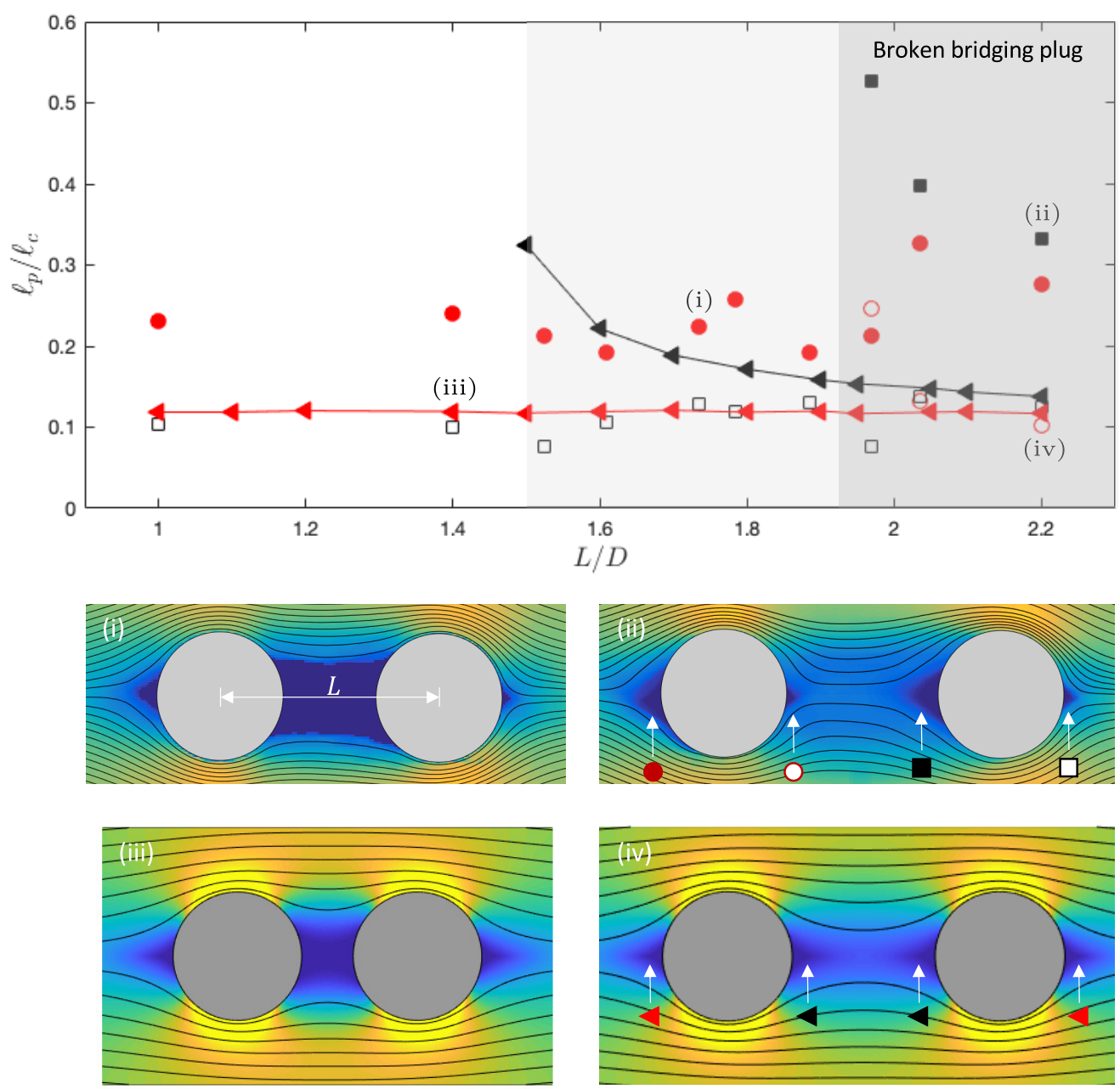

FIG. 9. Plug lengths for two disks aligned with the flow as a function of the center-to-center separation $L$, for experiments with untreated sheets and numerical computations; $B=2.7$ and $n=0.57$ (0.06\% Carbopol), The images (i)-(iv) below correspond to the separations identified in the main panel, with the symbols corresponding to the plug locations indicated, and show scaled speed maps (with color bars as in Fig. 4) and streamlines. Given the lack of any apparent trend with $L$, the scatter in the experimental data for the leading plug length (filled red circles), with a standard deviation of about $15 \%$, gives a sense of the overall uncertainty in the measurements.

obstacle must therefore cast a shadow on its follower, which increases the length of the front plug attached to that second obstacle just after the bridge is broken. These more quantitative details of the flow adjustments induced by the interaction of the obstacles are obscured by the scatter in the experimental measurements, but are more clearly identified in theoretical computations [see Fig. 9(b)]. Note that such interactions are very different from those occurring in unbounded flows around cylinders, where the yielded regions are localized to the obstacles and the surrounding plugs can sometimes (though not always) suppress any interaction [24].

Next, we conducted tests designed to constrain, in a different way, any microstructural ageing or relaxation: for the flow around an isolated disk, we varied the inlet flux $Q$ in a stepwise fashion over time and extracted the time-dependent plug length at the front of the disk. As seen in Fig. 10, 

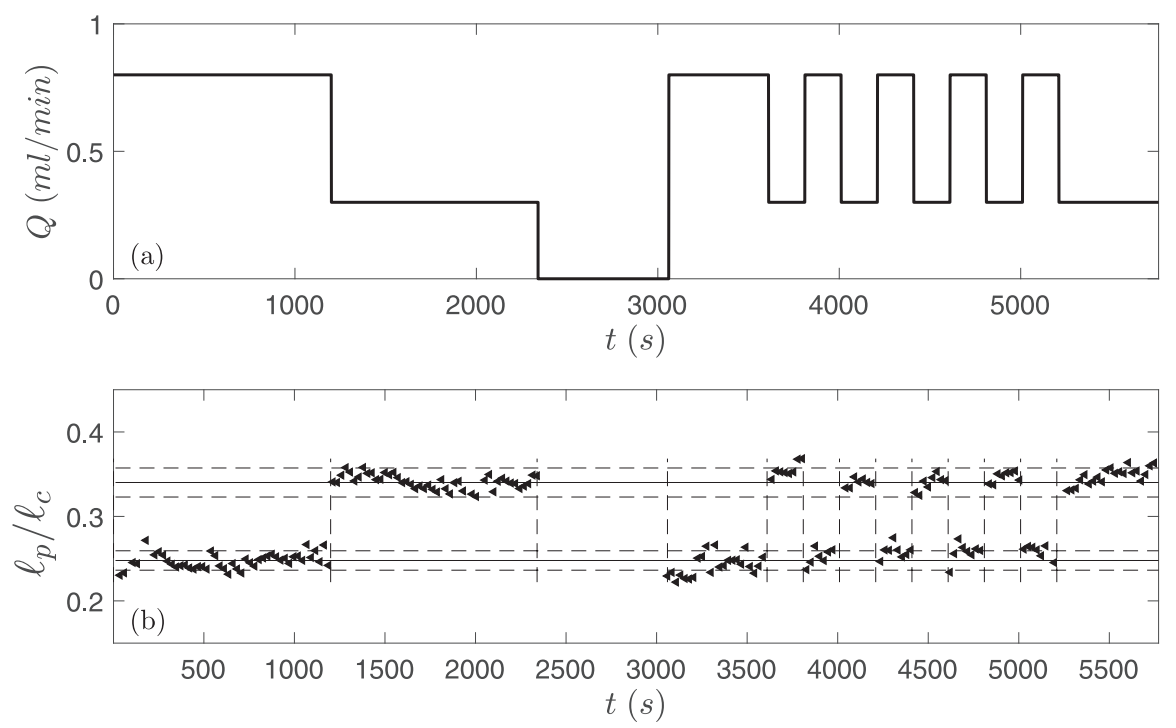

FIG. 10. Time dependency of the plug size on the front and back of an isolated disk subject to a flow rate $Q(t)$. The tests were performed with $0.06 \%$ Carbopol solution and treated sheets. The solid and dashed horizontal lines represent the average and standard deviation of the steady-state values reported in Fig. 7(b).

this length adopts its steady value after an undetectably rapid adjustment to the switch in flow rate. We repeated this test using a number of different protocols to increase or decrease the flow rate and found similar results. Thus, the characteristic relaxation time for rheological changes must be less than about $10 \mathrm{~s}$, the interrogation time for determining the plug size. This is consistent with both the lack of any larger-scale asymmetry in the flow patterns around separated disks and the absence of any noticeable hysteresis above the yield stress in the up-down flow curves of Fig. 2.

To emphasize this last point, we also prepared a different viscoplastic suspension by vigorously stirring $0.1 \% \mathrm{wt} / \mathrm{wt}$ Carbopol gel with a sharp blade at $1200 \mathrm{rpm}$ for around two and a half minutes, motivated by previous observations of the development of significant thixotropic behavior from a similar method of preparation [17]. Flow curves measured for this suspension did, indeed, exhibit significant hysteresis [Fig. 11(a)]. With this second type of Carbopol in the obstructed
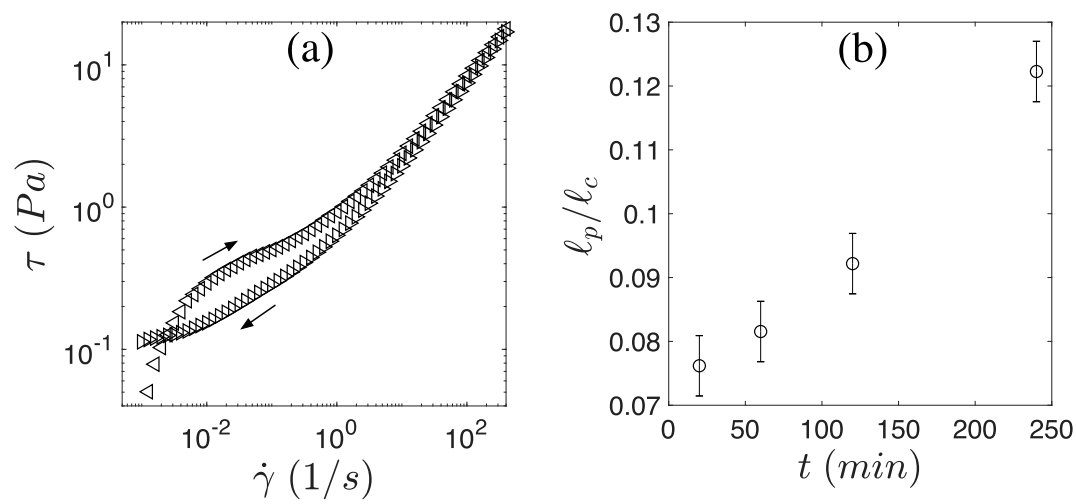

FIG. 11. (a) Thixotropic flow curves for an $0.1 \%$ Carbopol suspension that was very highly sheared during mixing (measured using the same rheometer as in Fig. 2, but with an up-down ramp controlling the shear rate). (b) Time series of the plug length after a step change in flow rate behind a circular obstruction in the Hele-Shaw cell using the same fluid (with flow rate $Q=0.05 \mathrm{ml} / \mathrm{min}$ after the step change). 
Hele-Shaw cell, the flow patterns were still fore-aft asymmetric, and the plug lengths were strongly time-dependent after step changes in flow rate [Fig. 11(b)]. In fact, experiments with this fluid were generally unreproducible and depended on the stress history of the fluid, in clear contrast to the main results reported in this paper. Similar unreproducible results and stress-history dependence were found when pumping Laponite (another widely documented thixotropic fluid) through the cell, further suggesting that thixotropy is not playing a significant role in the main results of this paper.

\section{DISCUSSION}

In this paper, we have provided an experimental study of the flow of a yield-stress fluid around obstructions in a thin slot. We also complemented these experiments with theoretical computations that generalize our previous analysis of flows of Bingham fluid in Hele-Shaw cells [13]. Both the experiments and theory demonstrate the appearance of stagnant plugs spanning the slot attached to the front and back of the obstacles, together with high-speed flows to either side. A number of trends observed in the experiments are successfully predicted by the theory. The theory also confirms an algorithm to estimate the size of the stagnant plugs attached to differently shaped obstacles, which may have practical application when controlling plug size is of importance. However, the experiments are strikingly different from the theoretical predictions in the fore-aft asymmetry of both the flow field and the size of the unyielded plugs. The asymmetry is not present in experiments with either a Newtonian fluid or, unlike in an unconfined geometry, a simple viscoelastic fluid. We find that the asymmetry is insensitive to effective slip over the walls of the slot and must be connected to some sort of rheological hysteresis of the Carbopol suspension that was used. The experiments suggest that the hysteresis arises over flow distances of a few millimeters given the typical flow rates through the cell $\left[O\left(10^{-4}\right) \mathrm{m} / \mathrm{s}\right]$, which translates to transit times of 10 or so seconds.

Carbopol suspensions have been observed to possess nonideal rheology in previous studies, either close to the yield stress or across a wider range of shear rates $[7,16,17,25]$. Nevertheless, the viscoelastic relaxation times expected for our Carbopol suspensions (given by the measurements of $G^{\prime}$ and $G^{\prime \prime}$ provided in Table I) are about a second or less. Thus, linear viscoelasticity below the yield stress does not appear to be capable of generating any fore-aft asymmetry, much as seen for an experiments conducted with a standard viscoelastic liquid (a PEO solution). Moreover, when we prepared a different suspension of Carbopol by vigorous mixing (Fig. 11), a pronounced thixotropy arose that significantly affected the flow patterns observed in the Hele-Shaw cell. However, the Carbopol that we used in most of our experiments exhibited no discernible hysteresis above the yield stress (Fig. 2), while still showing strong fore-aft asymmetry.

A key detail of viscoplastic Hele-Shaw flow is the flow structure across the cell: the yield stress establishes shear layers against the walls of the cell which border a central pluglike flow spanning the midplane of the slot (Fig. 1). Material inside that pseudoplug region is held slightly above the yield stress in order to accommodate the weak extension or shearing in the plane of the cell that permits flow around an obstruction [1,13]. This feature aside, strain rates and stresses in the plane of the cell are less significant than the shear rates and stresses across it. A significant fraction of the fluid across the slot is therefore always close to its yield stress throughout the quasi-two-dimensional flow pattern. Indeed, the thickness of the pseudoplug at any point is dictated by the local shear stresses across the slot, which can be directly inferred from the midplane velocity. The measurements in Fig. 4 imply that the pseudoplug thickens as fluid approaches the front of an obstacle, then thins more substantially as the material is swept through the high-speed regions at the top and bottom of the obstacle, before thickening once more in the wake. The lack of any noticeable hysteresis above the yield stress in the flow curves of the Carbopol suggests that relaxation over the shear layers is not responsible for the pronounced fore-aft asymmetry of the flow patterns. Instead, this asymmetry most plausibly arises from hysteresis near the yield stress, which becomes embedded in the erosion or growth of the central pseudoplug. However, further rheological work is required to identify the precise origin. 
[1] N. J. Balmforth, I. A. Frigaard, and G. Ovarlez, Yielding to stress: Recent developments in viscoplastic fluid mechanics, Annu. Rev. Fluid Mech. 46, 121 (2014).

[2] D. Bonn, M. M. Denn, L. Berthier, T. Divoux, and S. Manneville, Yield stress materials in soft condensed matter, Rev. Mod. Phys. 89, 035005 (2017).

[3] G. C. Vradis and A. L. Protopapas, Macroscopic conductivities for flow of Bingham plastics in porous media, J. Hydraul. Eng. 119, 95 (1993).

[4] S. H. Bittleston, J. Ferguson, and I. A. Frigaard, Mud removal and cement placement during primary cementing of an oil well-Laminar non-Newtonian displacements in an eccentric annular Hele-Shaw cell, J. Eng. Math. 43, 229 (2002).

[5] A. Roustaei and I. A. Frigaard, The occurrence of fouling layers in the flow of a yield stress fluid along a wavy-walled channel, J. Non-Newtonian Fluid Mech. 198, 109 (2013).

[6] A. N. Beris, J. A. Tsamopoulos, R. C. Armstrong, and R. A. Brown, Creeping motion of a sphere through a Bingham plastic, J. Fluid Mech. 158, 219 (1985).

[7] A. M. V. Putz, T. I. Burghelea, I. A. Frigaard, and D. M. Martinez, Settling of an isolated spherical particle in a yield stress shear thinning fluid, Phys. Fluids 20, 033102 (2008).

[8] D. L. Tokpavi, P. Jay, A. Magnin, and L. Jossic, Experimental study of the very slow flow of a yield stress fluid around a circular cylinder, J. Non-Newtonian Fluid Mech. 164, 35 (2009).

[9] Y. Holenberg, O. M. Lavrenteva, A. Liberzon, U. Shavit, and A. Nir, PTV and PIV study of the motion of viscous drops in yield stress material, J. Non-Newtonian Fluid Mech. 193, 129 (2013).

[10] O. G. Harlen, The negative wake behind a sphere sedimenting through a viscoelastic fluid, J. NonNewtonian Fluid Mech. 108, 411 (2002).

[11] I. Cheddadi, P. Saramito, B. Dollet, C. Raufaste, and F. Graner, Understanding and predicting viscous, elastic, plastic flows, Eur. Phys. J. E 34, 1 (2011).

[12] D. Fraggedakis, Y. Dimakopoulos, and J. Tsamopoulos, Yielding the yield-stress analysis: A study focused on the effects of elasticity on the settling of a single spherical particle in simple yield-stress fluids, Soft Matter 12, 5378 (2016).

[13] D. R. Hewitt, M. Daneshi, N. J. Balmforth, and D. M. Martinez, Obstructed and channelized viscoplastic flow in a Hele-Shaw cell, J. Fluid Mech. 790, 173 (2016).

[14] S. Drost and J. Westerweel, Hele-Shaw rheometry, J. Rheol. 57, 1787 (2013).

[15] G. Ovarlez and S. Hormozi (Eds.), Rheology of visco-plastic suspensions, Lectures on Visco-Plastic Fluid Mechanics (Springer, Cham, 2019), pp. 165-194.

[16] T. Divoux, C. Barentin, and S. Manneville, Stress overshoot in a simple yield stress fluid: An extensive study combining rheology and velocimetry, Soft Matter 7, 9335 (2011).

[17] M. Dinkgreve, M. Fazilati, M. M. Denn, and D. Bonn, Carbopol: From a simple to a thixotropic yield stress fluid, J. Rheol. 62, 773 (2018).

[18] S. Hormozi, D. M. Martinez, and I. A. Frigaard, Stable core-annular flows of viscoelastic fluids using the visco-plastic lubrication technique, J. Non-Newtonian Fluid Mech. 166, 1356 (2011).

[19] M. Christel, R. Yahya, M. Albert, and B. A. Antoine, Stick-slip control of the carbopol microgels on polymethyl methacrylate transparent smooth walls, Soft Matter 8, 7365 (2012).

[20] S. Jonas, D. Bhattacharya, M. K. Khokha, and M. A. Choma, Microfluidic characterization of cilia-driven fluid flow using optical coherence tomography-based particle tracking velocimetry, Biomed. Opt. Express 2, 2022 (2011).

[21] M. Daneshi, A. Pourzahedi, D. M. Martinez, and D. Grecov, Characterising wall-slip behavior of carbopol gels in a fully-developed Poiseuille flow, J. Non-Newtonian Fluid Mech. 269, 65 (2019).

[22] A. Poumaere, M. Moyers-González, C. Castelain, and T. Burghelea, Unsteady laminar flows of a carbopol gel in the presence of wall slip, J. Non-Newtonian Fluid Mech. 205, 28 (2014).

[23] E. Chaparian and I. A. Frigaard, Cloaking: Particles in a yield-stress fluid, J. Non-Newtonian Fluid Mech. 243, 47 (2017).

[24] E. Chaparian, A. Wachs, and I. A. Frigaard, Inline motion and hydrodynamic interaction of 2D particles in a viscoplastic fluid, Phys. Fluids 30, 033101 (2018).

[25] A. M. V. Putz and T. I. Burghelea, The solid-fluid transition in a yield stress shear thinning physical gel, Rheol. Acta 48, 673 (2009). 